\title{
A Learning-by-Doing Approach to Medication Management for Adolescent Diabetics
}

\author{
doi:10.3991/ijim.v3i1.701 \\ Steinar Kristoffersen \\ Østfold University College, Halden, Norway
}

\begin{abstract}
Many people suffer from potentially dangerous diseases. This paper is concerned with diabetes, in particular for young patients. Of course, most patients would agree that they need to worry about the risk of being taken ill in both the long and the short term. In principle, therefore, they are willing to change their behaviour according to medical stipulations. In the case of diabetes, this comprises measuring the level of glucose in the blood, observing a strict diet and exercising regularly. In practice, however, this turns out to be difficult for many patients. Making this a classical case of learning, the link from theory (knowing the mechanics of the disease) onto practice (changing the behaviour) needs to be strengthened. We propose a tool that may contribute to this, by explicating the dialogue between the learner and the "textbook model," which is an aggregate of medical expertise, parental supervision, personal experiences and the adaptation to the social and situated context. The tool is based on an explicit learning model, but the purpose is not one of promoting this particular model before the alternatives. The aim of this paper is to show how the tool can accommodate such models in general, and be used to further investigate their effectiveness in an empirical fashion. The selected model does, on the other hand, explicate a mobile and collaborative form of learning that may be useful in the context of treating adolescents with diabete
\end{abstract}

Index Terms —Mobility, adolescence, diabetics, learning, human computer interaction, quality of life

\section{INTRODUCTION}

Diabetes is a disease causing the body's own production of the insulin to be stopped or been greatly reduced. Insulin is necessary to allow cells to utilize sugar transported around the body through the blood. Without insulin, the blood sugar (glucose) level will continue to increase, causing various medical problems, and in the end be fatal to the patient. The treatment of diabetes consists of monitoring actual blood sugar level, and injecting insulin (typically using a syringe) several times a day. A diabetic may need to inject insulin before all meals as well as once or twice each day using a "long-term" type of insulin that provides a base insulin depot in the body. The amount of insulin that is injected is critical. Administration depends on establishing a "mental model" of the relationship between activity, food and medication, which is based on experience. Assessing the effect of food on blood sugar is non-trivial because different types of food contain different amounts of carbohydrates, and is transformed into blood sugar at very different rates.

A common objective will be to develop procedures that combine medical data, which are necessary or useful to adjust the dosage of drugs that comprise the cure, and patient parameters more generally. Different parameters will be requested depending on the illness, bearing in mind that we want our approach to be valid for other chronic diseases as well. Many of the parameters will be truly context-dependent, however, and their range and significance will in relevance and number vastly exceed the context-dependent data that we have seen alluded to for horizontal applications [19], e.g., blood sugar level and time of measurement, food intake and time of eating, physical exercise as well as time period and exercise level, heart rate, blood pressure and body temperature, crossmedication (even with non-prescription drugs), sleeping pattern, weight (loss) and body composition, etc. In the specification below, which illustrates our approach, the first three of these are explicated.

There are numerous challenges associated with the care of children and adolescents with diabetes. For instance, insulin doses may not easily be calculated on the basis of body weight, since individuals respond differently to hypoglycemic events (markedly low blood sugar levels) due to their variable hormone responses, which are different from adults [20]. The fear of hypoglycemia adds to the social and psychological challenges associated with the disease, and may cause less strict metabolic control [18]. Erring on the "safe side," however, may lead to recurring excessive levels of blood sugar, which is known to be a possible cause of eyesight and kidney failure, as well as other complications in the long term [8].

In terms of self-managing the disease, medial research presents results that are ominous, indeed:

"Health providers and family members may underestimate adolescents' difficulty managing hypo- and hyperglycemia appropriately. The presence of parental supervision does not ensure an appropriate response; parents may be particularly misinformed about the management of hyperglycemia [11, p. 171]."

The practice does not improve particularly as the patient grows older [2], which clearly indicates the need for a more deeply rooted approach to trying to change of behaviour through learning. The potential of mobile learning in such situations is great, as we know from other domains [3]. Continuous or timely responses to mundane challenges in patients' everyday life may be better suited and more convincing than traditional and theoretical approaches to teaching the patient about the management of diabetes and its consequences.

The research objective addressed in this paper is to explore a novel approach to technological support for learning for diabetes patients, by allowing them to explore and check the theoretical implication of their lived 
experiences. In our initial implementation, this is conceived as implementing a graphical user interface on top of a set of mathematical objects and equations, which enable advanced theorem checking by simple means.

\section{RELATED RESEARCH}

There have been several attempts made previously to develop mobile technology in aid of patients with diabetes. Some are still quite visionary, in terms of the advanced sensor technology that will be required in order for the patient to actually do the measuring themselves whilst mobile [15]. Other are already functional in an experimental setting, at least to the extent that they can be part of an empirical assessment [6]. Such systems all seem to have in common, however, that they are made to monitor or predict future blood sugar levels as accurately as possible, using a compilation of data collected by the patient.

There has been much work done previously in the area of insulin-dosage calculations algorithmically [16]. Pacini and Bergman use a model that they call the minimal model (MINMOD) to evaluate the metabolic control of glucose. The problem for our objectives of supporting children is that the input variables are most likely opaque to the patient, and even if they did, they would not be able to collect the data.

Islam et al. have expanded the minimal model with additional equations to implement an algorithm, which is still relatively simple, but comprises even more variables that have to be determined by professional doctors [10]. However, some of the insulin sensitivity parameters can also be defined by studying computer simulated glucose profiles and this is an opportunity that needs to be considered. Another algorithm proposed by Fisher, is more adapted to a situation such as ours [5]. It is still based, however, on a much too theoretical analysis of the control of blood glucose level and the interaction between the glucose and insulin. It is based on the presence of three plasma glucose samples and although the author acknowledges that the meals are most important for predicting the glucose levels, it does not pragmatically consider what sort of food a diabetic is eating. Without knowing (by heart) the glycemic index of a composite meal, which is almost impossible to do manually even if a table of glycemic indices for each ingredient were available, it would be difficult to give a prediction of the individual response.

Ubiquitous monitoring and control of glucose levels and insulin dosages seems to be ideal for this condition, and intuitively fruitful, but the observable effects are actually usually quite negligible [13]. The results may point in the right direction, but the reduction in average blood glucose levels is not yet found to be statistically significant [4]. On the other hand, taking a wider perspective comprising motivation factors, have been found to improve the self-management, e.g., in one case of embedding it in a game context [14].

Based on the reasoning above, we have decided to look at the administration and management of enduring medical conditions in a mobile learning perspective. The need to develop a specific curriculum for diabetes patients, has previously been recognized [12]. Previous work also exists, which has attempted to make this curriculum ubiquitously available as an instrument of learning as well as intervention [24]. This experiment uniformly produced encouraging and statistically significant improvements in levels of glucoses, competencies and perceived quality of life.

Endeavours such as the one described above, may be seen as excessively resource consuming, however, since they involve making an ambulant team of human experts available to the patients and their families. Our aim is different, inasmuch as we seek to develop constantly available mechanisms, which will allow for explorative learning from patient-specific and context-aware simulations of the insulin-glucose reaction. Instead of human expertise, we want to experiment with the facilitation of direct interaction with a computer simulation. We see this as a "boundary object [7]," which may mediate and translate the requirements, requests and responses between patients, parents, carers and medical expertise.

Recognizing the need to support intervention in order to change behaviour, rather than expecting learning to come straightforwardly from the patient's exposure to future predictions, is an important novel aspect of the project that is described in this paper. Another, which is related, is that instead of making one prediction, which is as exact as possible, we encourage the user to explore the complete state space on the basis of more easily compiled and approximate input. Thus is can more easily be carried out, which will in itself, we hope encourage adoption of the system.

Hence, a technological aid is proposed below, in order to make it easier to collect and compile the necessary data. Taking the theoretically informed approach of designing intervention by a so-called "boundary-object", we hope to find exactly the combination of aspects that optimized behavioural change (observable learning), whilst minimizing the need for complicated measuring regimes.

\section{IMPLEMENTING A BOUNDARY OBJECT}

It seems clear that in order to work properly under the conditions that we have outlined above, a device needs to satisfy several requirements. It needs to be robust and easy to use. It has to be sufficiently fast and power efficient. However, we need to go beyond that in order to arrive at a solution that will work for many diverse groups, and in rough and unwelcoming physical conditions as well as socially, and can tie together the concerns of multifarious user groups, such as the parents of a kid with diabetes on one hand, and his friends on the other.

Dedicated devices, which serve specialist-functions, are of course one category of medical equipment that we need to look at, but considering that we are targeting children, this cannot be the limit of our scope. We need to look at the opportunities that exist to integrate heterogeneous devices in order to increase the capacity of cell phonebased healthcare solutions. Therefore we are also going to try to "make medical" much simpler and more available equipment, such as sports' heart-rate monitors, by inputting their data to the mobile phone application that we propose to develop. Such devices are precise [23], robust and readily available where more advanced medical apparatus is not.

For the reasons sketched above, we have looked at the notion of a boundary object as one way of informing the design of a tool that can compile and enforce a sufficiently 
strict administrative regime for medical cure adherence. For now, we are going to keep our scope to the harnessing of diabetes with insulin, with particular concern for children since they are less likely to keep track of nutrition, level of activity and insulin dosages themselves.

In previous IS (Information Systems) literature, the notion of boundary objects has had mainly analytical purchase [22]. Therefore, it is a novel contribution of this paper to draw inferences from the usage of the concept for design purposes. There is very little literature on boundary objects as a design component, except "after-the-fact" as one discovers the role such objects have come to play [9]. Instead, what we are going to try to do in this paper is to use it as a proactive means of eliciting the design ideas (rather than only mediating them).

The problem that we face in designing a smart tool for our purposes is similar to the one described by Star and Griesemer (1989), in particular because the internalization of the algorithm, which models insulin's role in the metabolism, e.g., is such an integral part to actually using it. Learning and motivation goes hand in hand, and the boundary object as a perspective on knowledge therefore becomes very useful.

"The creation of scientific knowledge depends on communication as well as on creating new findings. But because these new objects and methods mean different things in different worlds, actors are faced with the task of reconciling these meanings if they wish to cooperate [22] “

In the terminology of Star and Griesemer, actors inhibit different social worlds, and when they encounter the knowledge interest of parties "from elsewhere" they reinterpret their agenda to fit better with their own. They then try to control action, which is related to the agenda through the object. This is the process of translation. As a concept, it fits with our selected domain, which is populated by parents, doctors and children with diabetes. Doctors need to treat many patients efficiently, and look twice only at the exceptional situations. The parents will seek stability and predictability for all family members, while the average kid with diabetes is more likely to want to live life just like his or her friends. Doctors and parents can use coercion to maintain the dominant perspective of their own interpretation of the situation [7], but this option is generally quite limited. In general, the dominant "gatekeeper" or any given "obligatory point of passage" must take into account the interests of all parties. In the next paragraphs we are going to outline the design of a gatekeeper, by means of a boundary object.

A boundary object is an analytical conceptualization of phenomena that "inhibit several intersecting social worlds [22]. Boundary objects cannot be static or standardized, in the sense that there must be room for them to fill different informational needs in different social worlds. The have to be similar enough to be recognizable as a means of translation across social worlds, however, and

"The creation and management of boundary objects is a key process in developing and maintaining coherence across intersection social worlds [22]."

We want to focus on the learning aspects. We want to think about it in terms of simple parameters, which can be estimated or measured relatively accurately by the patient. We start with levels of activity, along a scale of perceived exertion/effort. Next we want to include a measure of the blood sugar levels, which is absolutely essential for a sufficiently precise administration of insulin. Finally, the nutritional components have to be assessed, e.g., by breaking down by their glycemic indices the components of meals to help the patient anticipate which levels of activity or insulin dosages may become necessary soon. The task is now to make a model with which interaction can be accurately modelled, and then see if it can be implemented to satisfy the requirements of a boundary object.

Our proposal is to make an executable specification, which we have called CATLA (Computerized Aid for Therapeutic Learning in Action), as the first step in the project. We use the declarative programming language Maude (http://maude.cs.uiuc.edu/). The detailed characteristics of the language are outside the scope of this paper. We focus only on the so-called rewriting rules.

Consider, for instance, the rule labeled [activity ], which emulates a change in the activity level of the patient and generates an auxiliary message about it to the rest of the system, which can then respond appropriately.

We have specified a module, which (edited for readability) looks like the following:

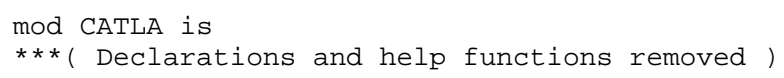




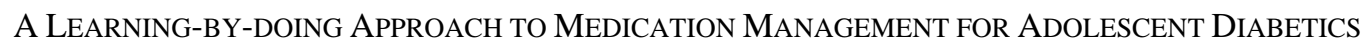

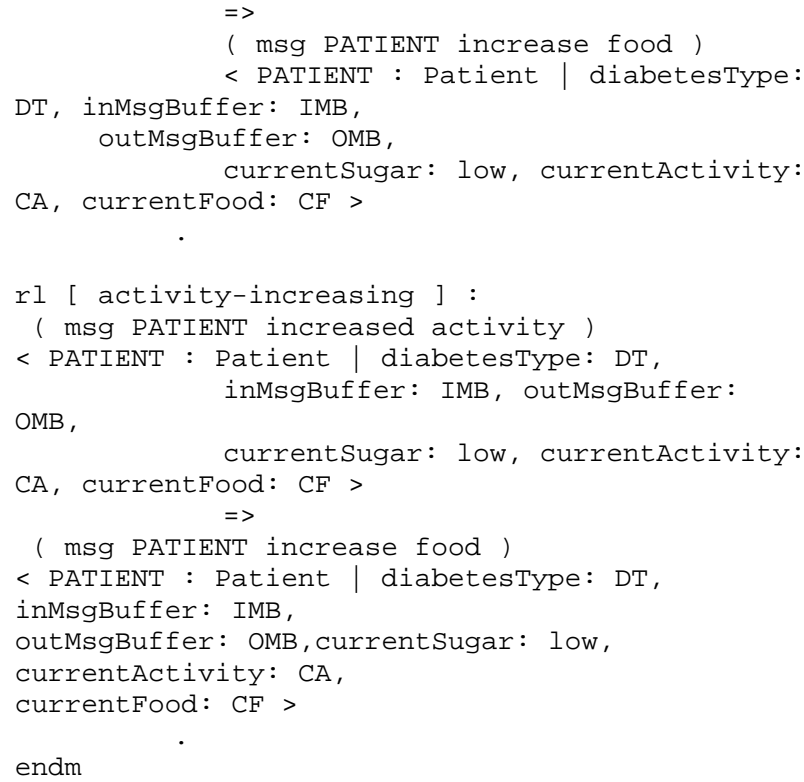

For the purpose of illustration in this paper, the two main rewriting rules are [ sugar-too-low ] and [ activity-increasing ]. The former makes sure that a patient always is told to increase his food intake (which may mean taking pure sugar or fruit juice) to acutely treat a situation which comprises dangerously low blood sugar level. The latter makes sure that we simulate the same instruction given to a patient as a result of self-reporting that the level of activity is increasing. The outcome is the same, but the causes are different. We can then run a simulation asking the Maude interpreter to find a state in which the patient was instructed to take in more food. The interpreter will according to the specified criteria search the entire state space (if it is finite), and list the number of matching states that we asked for.

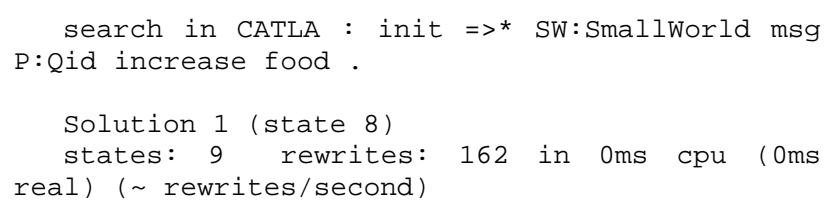

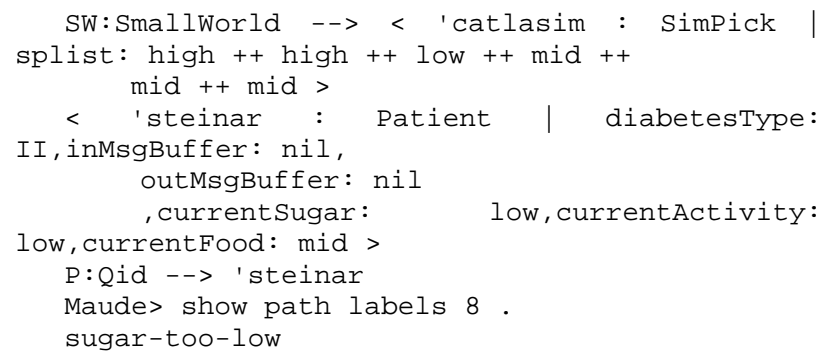

Showing the path which lead to the state concludes that the reason was that the blood sugar level had dropped too low.

The current implementation is much to rudimentary to be used as an exact vehicle of future blood sugar level prediction. At the same time it is a far too mathematical for any patient to be able to relate to. It is, we admit, the mathematical qualities of the object, which on the other hand makes model checking and exploration possible that also makes it pretty useless in practice, as it stands now. Arbitrarily exact and complex models may of course be implemented, which is encouraging in terms of future work and prototyping for actual patients, but in terms of this being a "boundary object", which can be internalized and used by these patients, that will only exacerbate the situation. A better user interface is obviously needed. The next section clarifies this part of the system, in order to support its aggregation into a proper boundary object.

\section{THE MODEL}

In order to structure the user interface, this paper is inspired by the notion of a "learning model". The intention is not to enforce learning according to this particular model, which we have no reason to believe would be more efficient than any other model of a similar kind. However, a case such as ours provides some opportunities to observe a pattern of use emerging, as it can be logged over time. This means that the model can be empirically assessed and improved throughout the project. As such it may be seen simply as a hypothesis about how the "learning for life" scenario through the user interface, conceivably may be presented. The top levels of the model can be illustrated by the figure below [21]:

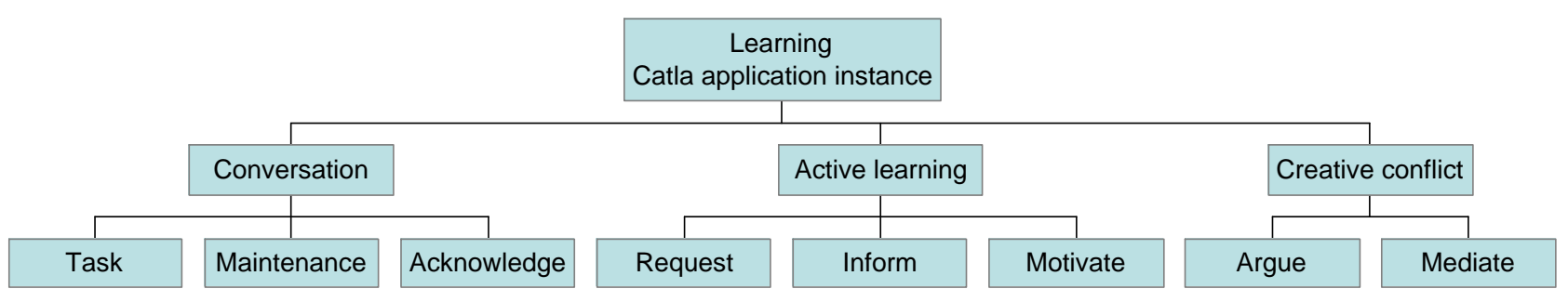

Figure 1. The collaborative learning process 
TABLE I.

THE LEAF NODES OF THE COLLABORATIVE LEARNING MODEL

\begin{tabular}{|c|c|c|c|c|c|c|c|}
\hline Task & Maintenance & Acknowledge & Request & Inform & Motivate & Argue & Mediate \\
\hline $\begin{array}{l}\text { Co-ordinate } \\
\text { group process }\end{array}$ & Request attention & Appreciation & Information & Rephrase & Encourage & Conciliate & $\begin{array}{l}\text { Teacher } \\
\text { mediation }\end{array}$ \\
\hline $\begin{array}{l}\text { Request fo-cus } \\
\text { change }\end{array}$ & Suggest action & Accept/ Confirm & Elaboration & Lead & Reinforce & Agree & \\
\hline $\begin{array}{l}\text { Summarize } \\
\text { information }\end{array}$ & $\begin{array}{l}\text { Request } \\
\text { confirmation }\end{array}$ & Reject & Clarification & Suggest & & Disagree & \\
\hline \multirow[t]{5}{*}{$\begin{array}{l}\text { End } \\
\text { participation }\end{array}$} & Listening & & Justification & Elaborate & & $\begin{array}{l}\text { Offer } \\
\text { alternative }\end{array}$ & \\
\hline & \multirow[t]{4}{*}{ Apologize } & & Opinion & $\begin{array}{l}\text { Explain/ } \\
\text { Clarify }\end{array}$ & & Infer & \\
\hline & & & Illustration & Justify & & Suppose & \\
\hline & & & & Assert & & $\begin{array}{l}\text { Propose } \\
\text { exception }\end{array}$ & \\
\hline & & & & & & Doubt & \\
\hline
\end{tabular}

We realize that the leaf nodes of the model, which are presented in the table above, may be too generic for our purposes, and that one distinguishable objective in the near future will be to "instantiate" each of them with terminology that overlaps with the specific use context that our application targets. The current wording is not likely to make sense to our subjects. However, as a "neutral" starting point, we have kept the terminology in the first version, in order to keep a stable terminology making tracking and re-design easier until a proper evaluation with real users can be carried out.

\section{THE USER INTERFACE}

The pertaining interaction design is something that we believe can be pursued in several planned steps within the project that we have outlined in this paper, in order to develop the specification into a boundary object that can aid the learning of appropriate strategies for diabetes patient which have to relay on simple and mobile, yet "smart" technology. One of the most important aspects of such a model would be to learn to live, and to live well, with being treated under a strict and permanent medical course of therapy.

The application comprises a simple mechanism for storing observations, which is a textual database. It is not a computationally optimized format, in any sense, but simply a straightforward way of embedding this information and the search clause of a Maude specification. It would typically look something like the following:

< 'steinar : Metric | timestamp: Fri Apr 25 $15: 17: 22$ CEST 2008

exercise: No exercise, carbs: No carbs, insulin: Middle insulin >

< 'steinar : Metric | timestamp: Fri Apr 25

$15: 18$ : $\odot \odot$ CEST 2008

, exercise: High exercise, carbs: No carbs, insulin: No insulin >

< 'steinar : Metric | timestamp: Fri Apr 25 $15: 19$ : $\odot 8$ CEST 2008

exercise: No exercise, carbs: No carbs, insulin: High insulin >

The specification of the query is, however, not textual like above, but deducted from the application of the learning model described above, at the graphical user interface.

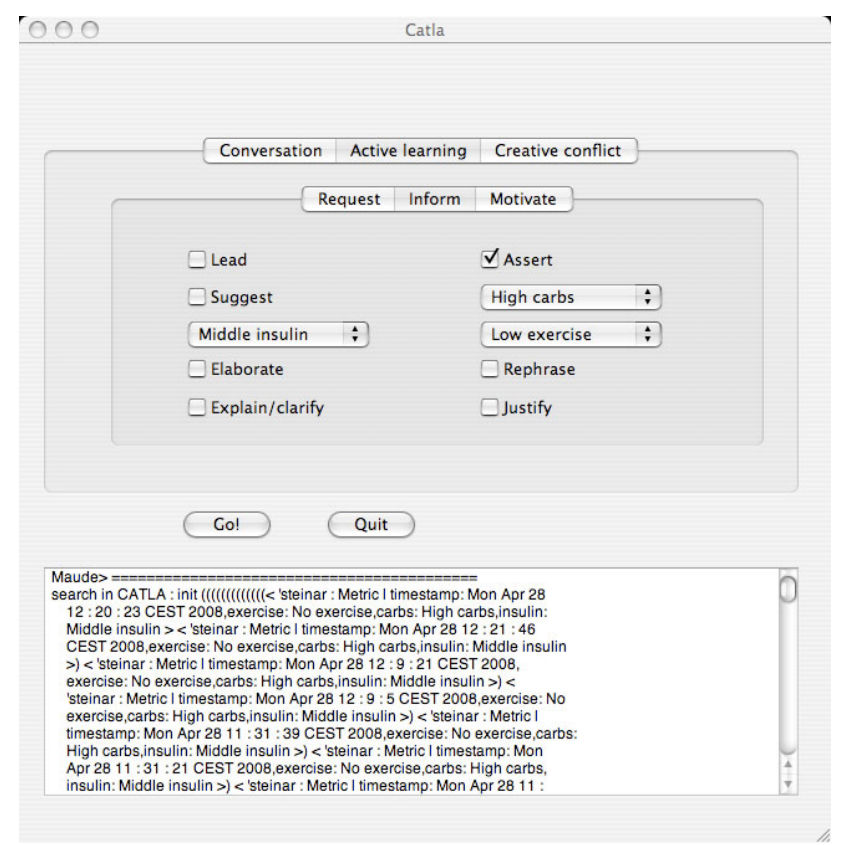

Figure 2. The user interface of CATLA

The user is intended to be looking up any of the tabviews, which comprise the categories corresponding to the higher levels of the learning model. The leaf nodes are accessed/addressed by ticking them off in the forms underneath. Any combination is possible, because the client translates them individually to messages in Maude, for the CATLA application to depart from when the state space is then explored. And this is a core aspect of this application, as it currently stands. It simulates not only the development, admittedly even that in a very rudimentary fashion, of the increase of blood sugar following variation of exercise levels and carbohydrate intake, or the pancreatic response to it. It also simulates the collaborative learning environment, based on an explicit model. The assessment of the learning model in future work is going to explore it capability to contribute to the motivation and change of behavior of the patient, by allowing it to be a vehicle of medically informed exploration of the body's physiological response. 
The contribution to interaction design of the boundary object, is to provide an aggregate of three distinct perspectives, each firmly rooted in four different professional domains:

1. Medical competencies concerning the pancreatic response to variations in exercise levels, carbohydrate intake, and insulin administration.

2. The pedagogical insight that is embedded in the collaborative learning model, and which be believe may be strengthened even further by the systematic assessment that we make possible through implementing it in CATLA.

3. Algebraic specification of the dynamic properties of the entire state space possible from a given initial condition. It can show the invariants governing the system, and allows a systematic and definitive exploration of its properties, relative to the current patient.

4. Interaction design, which is initially very rudimentary with regards to the approach to learning. On the other hand, it takes a long step in adapting a graphical user interface as such to the underlying infrastructure, so that the algebraic exploration is possible to begin with.

The presentation of the results from the queries is still rather simple, in the form of a textual response from the Maude interpreter. User friendliness, as such, is beyond the scope of the paper, however, which is to show that, albeit still raw in terms of usability, the boundary object constitutes a proper tool chain, which can be summarized in the diagram below (Figure 3):

Thus, the contribution of this paper is to demonstrate a prototype of a boundary object comprising physiological aspects, learning science and interaction design, onto a mathematical object by which the patient can then explore his or her own insulin responses according to a provably correct model. It is not intended to be more physiologically "precise" than previous predicationoriented models, but it is our aspiration that it will to a larger extent contribute to changing patients' behaviors. For current state-of-the-art, this is where the expectations have failed to be met.

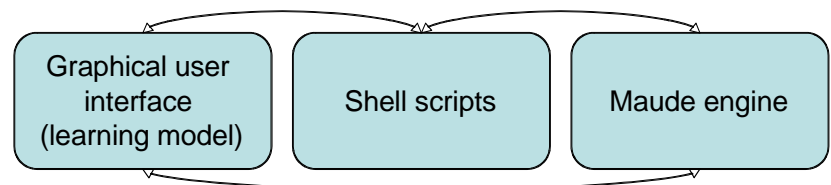

Figure 3. The tool chain architecture

\section{CONCLUSION}

This is an early step towards designing an aid to people who have previously been underserved in terms of technological support for adherence to longitudinal medication. Many types of medical treatment are given as longitudinal cures comprising a combination of drugs. The amount, time and type of drugs that need to be administered may rely on many different factors, such as the stage of the illness, height and weight, the patients' level of activity or recent food intake, as well as interaction with other drugs. Numerous patients struggle to keep up with their cures, e.g., children, elders and patients in lesser-developed areas. Failure to adhere to the administration plan for longitudinal treatment of acute illnesses is often critical to the outcome of the cure [1]. We would like in particular to address the issues related to quality of life and outcome expectations from longitudinal cures for children.

Existing technology is generally too heavyweight and expensive, too intrusive and demand data that mobile users are not in a position to get. We have suggested to see the notion of this technology as a boundary object, in order to find ways around these practical obstacles, and at the same time setting ourselves up to provide a more pedagogical presentation of the algorithm which prescribes how much and when to take insulin, adjust the activity level or take in more nutrition.

We have seen some examples recently that mobile technology combined with the multifarious perspectives of friends and fellow users, medical consultants etc., have been established as normative and effective implementations in this respect ${ }^{1}$. Some of these systems are proven in practice [17]. Our project starts from the understanding that such approaches are instrumentally sound, but we wish in addition to develop a pedagogical element on top of that. Thus, the next step will be to assess with users whether the "boundary object" in our guise of a running Maude specification can be used as a way of learning more and better how to live well under longitudinal administration of drugs. This will give us opportunities to explore the pedagogical outcome of the logical properties of the specification. We hypothesize that the assisted executions of search strategies on basis of the specification are going to confirm in principle its potential as a boundary object. It may have to be reimplemented on top of a user-friendlier platform, in order for the specification to become a fully functional and "live" boundary object, which actually makes living with diabetes easier. This is the ultimate goal of this project, of course, but in terms of verifying the correctness of the underlying model, Maude is a promising tool.

\section{ACKNOWLEDGMENT}

I would like to thank the reviewers of the mLearn 2008 conference for their helpful comments.

\section{REFERENCES}

[1] L. Atkins and L. Fallowfield, "Intentional and non-intentional non-adherence to medication amongst breast cancer patients," European Journal of Cancer, Volume 42, Issue 14, Pages vol. 42, pp. 2271-2276, 2006.

[2] M. Balfe, "Diets and discipline: the narratives of practice of university students with type 1 diabetes," Sociology of Health \& Illness, vol. 29, pp. 136-153, 2007.

[3] G. Connor, B. Peter, H. Steve, B. James, B. Nicole, and B. Ron, "'heh - keeps me off the smokes...": probing technology support for personal change," in Proceedings of the 20th conference of the computer-human interaction special interest group (CHISIG) of Australia on Computer-human interaction: design: activities, artefacts and environments Sydney, Australia: ACM, 2006.

[4] A. J. Farmer, O. J. Gibson, C. Dudley, K. Bryden, P. M. Hayton, L. Tarassenko, and A. Neil, "A Randomized Controlled Trial of the Effect of Real-Time Telemedicine Support on Glycemic Control in Young Adults With Type 1 Diabetes (ISRCTN 46889446)," Diabetes Care, vol. 28, pp. 2697-2702, November 1, 20052005.

\footnotetext{
${ }^{1}$ http://www.packitin.org/
} 
[5] M. E. Fisher, "A semiclosed-loop algorithm for the control of blood glucose levels in diabetics," Biomedical Engineering, IEEE Transactions on, vol. 38, pp. 57-61, 1991. (doi:10.1109/10.68209)

[6] D. Gammon, E. Årsand, O. Walseth, N. Andersson, M. Jenssen, and T. Taylor, "Parent-Child Interaction Using a Mobile and Wireless System for Blood Glucose Monitoring," Journal of medical Internet research vol. 7, 2005.

[7] S. Gasson, "Boundary-Spanning Knowledge-Sharing In ECollaboration," in Proceedings of the 38th Annual Hawaii International Conference on System Sciences(HICSS'05) 2005, pp. 245-.

[8] J. N. Harvey and B. Allagoa, "The long-term renal and retinal outcome of childhood-onset Type 1 diabetes," Diabetic Medicine, vol. 21, pp. 26-31, 2004. (doi:10.1046/j.1464-5491.2003.01062.x)

[9] K. Henderson, "Flexible Sketches and Inflexible Data Bases: Visual Communication, Conscription Devices, and Boundary Objects in Design Engineering," Science Technology Human Values, vol. 16, pp. 448-473, October 1, 19911991.

[10] S. Islam, J. Leech, C. C. Y. Lin, and L. Chrostowski, "Peak Blood Glucose Prediction Algorithm Following a Meal Intake," in Electrical and Computer Engineering, 2007. CCECE 2007. Canadian Conference on, 2007, pp. 579-582.

[11] S. Johnson, A. Perwien, and J. Silverstein, "Response to hypo- and hyperglycemia in adolescents with type I diabetes.," J Pediatr Psychol, vol. 25, pp. 171-8, April-May 2000. (doi:10.1093/jpepsy/25.3.171)

[12] J. Knowles, H. Waller, C. Eiser, S. Heller, J. Roberts, M. Lewis, K. Wilson, T. Hutchinson, M. Willan, P. Bavelja, G. Bennet, and K. Price, "The development of an innovative education curriculum for 11-16 yr old children with type 1 diabetes mellitus (T1DM)," Pediatric Diabetes, vol. 7, pp. 322-328, 2006. (doi:10.1111/j.1399-5448.2006.00210.x)

[13] A. Kollmann, M. Riedl, P. Kastner, G. Schreier, and B. Ludvik, "Feasibility of a Mobile Phone-Based Data Service for Functional Insulin Treatment of Type 1 Diabetes Mellitus Patients," Journal of Medical Internet Research, vol. 9, 2007.

[14] V. S. Kumar, K. J. Wentzell, T. Mikkelsen, A. Pentland, and L. M. Laffel, "The DAILY (Daily Automated Intensive Log for Youth) Trial: A Wireless, Portable System to Improve Adherence and Glycemic Control in Youth with Diabetes," Diabetes Technology \& Therapeutics, vol. 6, pp. 445-453, 2004. (doi:10.1089/152091 5041705893)

[15] A. G. Mathews and R. Butler, "A vision for the use of proactive mobile computing tools to empower people with chronic conditions," in Computer-Based Medical Systems, 2005. Proceedings. 18th IEEE Symposium on, 2005, pp. 425-427.

[16] G. Pacini and R. Bergman, "MINMOD: a computer program to calculate insulin sensitivity and pancreatic responsivity from the frequently sampled intravenous glucose tolerance test," Comput
Methods Programs Biomed, vol. 23, pp. 113-22, 1986. (doi:10.1016/0169-2607(86)90106-9)

[17] J. Prochaska, W. Velicer, C. Redding, J. Rossi, M. Goldstein, J. DePue, G. Greene, S. Rossi, X. Sun, J. Fava, R. Laforge, W. Rakowski, and B. Plummer, "Stage-based expert systems to guide a population of primary care patients to quit smoking, eat healthier, prevent skin cancer, and receive regular mammograms.," Prev Med. , vol. 41, pp. 406-16, August 2005. (doi:10.1016/j.ypmed.2004.09.050)

[18] C. M. Ryan and D. J. Becker, "HYPOGLYCEMIA IN CHILDREN WITH TYPE 1 DIABETES MELLITUS: Risk Factors, Cognitive Function, and Management," Endocrinology \& Metabolism Clinics of North America, vol. 28, pp. 883-900, 1999. (doi:10.1016/S0889-8529(05)70107-9)

[19] A. Schmidt, M. Beigl, and H.-W. Gellersen, "There is more to context than location," Computers and Graphics, vol. 23, pp. 893901, 1999. (doi:10.1016/S0097-8493(99)00120-X)

[20] J. Silverstein, G. Klingensmith, K. Copeland, L. Plotnick, F. Kaufman, L. Laffel, L. Deeb, M. Grey, B. Anderson, L. A. Holzmeister, and N. Clark, "Care of Children and Adolescents With Type 1 Diabetes: A statement of the American Diabetes Association," Diabetes Care, vol. 28, pp. 186-212, January 1, 20052005.

[21] A. Soller, "Supporting social interaction in an intelligent collaborative learning system," International Journal of Artificial Intelligence in Education, vol. 12, pp. 40-62, 2001.

[22] S. L. Star and J. R. Griesemer, "Institutional Ecology, 'Translations' and Boundary Objects: Amateurs and Professionals in Berkeley's Museum of Vertebrate Zoology, 1907-39," Social Studies of Science, vol. 19, pp. 387-420, Aug. 1989. (doi:10.1177/030631289019003001)

[23] F. Treiber, L. Musante, S. Hartdagan, H. Davis, M. Levy, and W. Strong, " Validation of a heart rate monitor with children in laboratory and field settings.," Medical Science Sports Exercise, vol. 21, pp. 338-342, June 1989. (doi:10.1249/00005768198906000-00019)

[24] S. von Sengbusch, E. Muller-Godeffroy, S. Hager, R. Reintjes, O. Hiort, and V. Wagner, "Mobile diabetes education and care: intervention for children and young people with Type 1 diabetes in rural areas of northern Germany," Diabetic Medicine, vol. 23, pp. 122-127, 2006. (doi:10.1111/j.1464-5491.2005.01754.x)

\section{AUTHORS}

S. Kristoffersen is with Østfold University College, N1757 Halden, Norway (e-mail: sk@ hiof.no).

This article was modified from a presentation at the mLearn2008 conference in Wolverhampton, Great Britain, October 2008. Manuscript received 23 October 2008. Published as submitted by the author. 